\title{
The origins of diffuse and globular organics in chondrites and IDPs revealed by nanobeam techniques
}

\author{
CHRISTIAN VOLLMER ${ }^{1}$, JAN LEITNER ${ }^{2}$, DEMIE \\ KEPAPTSOGLOU ${ }^{3}$, QUENTIN M. RAMASSE ${ }^{3}$, ASHLEY J. \\ $\mathrm{KING}^{4}$, PAUL F. SCHOFIELD ${ }^{4}$ AND HENNER \\ BUSEMANN $^{5}$ \\ ${ }^{1}$ Institut für Mineralogie, Universität Münster \\ ${ }^{2}$ Max Planck Institute for Chemistry \\ ${ }^{3}$ SuperSTEM Laboratory \\ ${ }^{4}$ Natural History Museum \\ ${ }^{5}$ ETH Zürich \\ Presenting Author: christian.vollmer@wwu.de
}

The functional chemistry and isotopic composition of organic matter (OM) within carbonaceous chondrites and interplanetary dust particles (IDPs) allow to study nebular and parent body processes in the early Solar System and the origins of biomolecules on early Earth [e.g., 1-3]. The Renazzo-type (CR) chondrites are of particular interest in this respect, because OM within some of these complex breccias is only minimally altered by fluid reactions [4]. Furthermore, comparison of functional chemistry data obtained by low-kV Scanning Transmission Electron Microscopy - Electron Energy Loss Spectroscopy (STEM-EELS) with data analyzed by Scanning Transmission Xray microscopy (STXM) helps to disentangle fine-scale heterogeneities and to control OM damage [5]. Such an approach is less representative than bulk analyses on extracted OM, but provides important information on unprocessed grains within their petrographic context. The combination of these techniques has, for example, recently shown that the $\mathrm{CM}$ chondrite Maribo contains primordial ${ }^{15} \mathrm{~N}$-depleted $\mathrm{OM}\left(\delta^{15} \mathrm{~N}_{\text {air }} \sim-200 \%\right)$ occurring as submicron organic aggregates dispersed within the matrix [6]. Their C-N functional chemistry shows typical signatures of extraterrestrial $\mathrm{OM}$ with varying intensity on a nm scale. In this ongoing project, we have analyzed C-N K-edge chemical data obtained by STXM on CR OM previously analyzed by EELS [4] and on diffuse cometary ${ }^{15} \mathrm{~N}$-enriched IDP OM. Whereas nitrogen K-edge data by EELS is usually rather noisy and sometimes difficult to assign to certain bonding environments, signal-to-noise quality of STXM data is more distinct at the expense of spatial resolution. In general, Renazzo OM exhibits a complex functional makeup among various ${ }^{15} \mathrm{~N}$ enriched "hotspots", which is similar to the small-scale heterogeneity observed within a single grain.

Acknowledgements: The DFG is acknowledged for funding this project in the course of the SPP 1833.

References : [1] Alexander C.M.O'D. et al. (2017), Chemie der Erde 77 (2), 227. [2] Busemann H. et al. (2006), Science 312, 727. [3] Rotelli L. et al. (2016), Sci. Rep. 6, 38888. [4] Vollmer C. et al. (2020), Meteorit. Planet. Sci. 55(6), 1293. [5] Stroud R. M. et al. (2021), Lun. Planet. Sci Conf. 52, abstr. \#2745. [6] Vollmer C. et al. (2020), Sci. Rep. 10, 20251. 